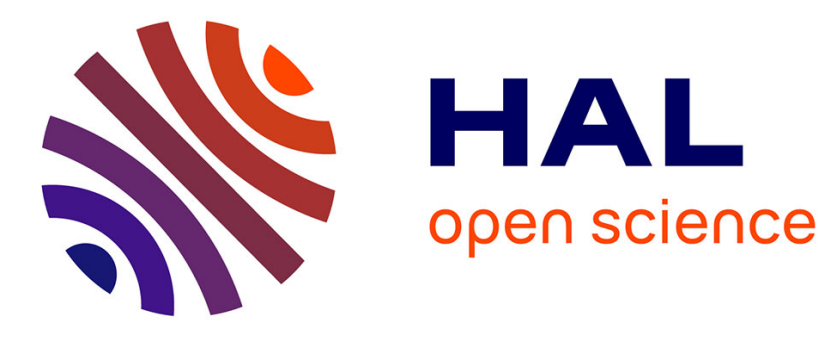

\title{
First Steps toward Location of Landmarks on X-Ray Images
}

\author{
Michel Desvignes, Barbara Romaniuk, Régis Clouard, Ronan Demoment, \\ Marinette Revenu, Marie-Josèphe Deshayes
}

\section{- To cite this version:}

Michel Desvignes, Barbara Romaniuk, Régis Clouard, Ronan Demoment, Marinette Revenu, et al.. First Steps toward Location of Landmarks on X-Ray Images. 15th International Conference on Pattern Recognition, 2000, Barcelone, Spain. pp.275-278, 10.1109/ICPR.2000.906066 . hal-00843450

\author{
HAL Id: hal-00843450 \\ https://hal.science/hal-00843450
}

Submitted on 12 Jul 2013

HAL is a multi-disciplinary open access archive for the deposit and dissemination of scientific research documents, whether they are published or not. The documents may come from teaching and research institutions in France or abroad, or from public or private research centers.
L'archive ouverte pluridisciplinaire HAL, est destinée au dépôt et à la diffusion de documents scientifiques de niveau recherche, publiés ou non, émanant des établissements d'enseignement et de recherche français ou étrangers, des laboratoires publics ou privés. 


\title{
First Steps toward Automatic Location of Landmarks on X-Ray Images
}

\author{
M. Desvignes, B. Romaniuk, R. Clouard, R. Demoment, M. Revenu, MJ. Deshayes \\ GREYC-CNRS 6072, 6 bd maréchal juin, 14050 CAEN; FRANCE \\ E-mail : Michel.desvignes@greyc.ismra.fr
}

\begin{abstract}
We address the problem of locating some anatomical bone structures on lateral cranial X-Ray Images. These structures are landmarks used in orthodontic therapy. The main problem in this pattern recognition application is that the landmarks are difficult to distinguish on images even for the human expert, because of lateral projection of the X-Ray process. We propose a 3 steps approach: the first step provides a statistical estimation of the landmarks, using an adaptative coordinates space. The second step computes a region of interest around the estimated landmark. In the third step, each landmark is precisely located using its anatomical definition. This paper describes the two first generic steps, and gives examples of the last step for two anatomical points.
\end{abstract}

\section{Introduction}

The goal of orthodontic and orthognatic therapy is to improve the interrelationships among craniofacial tissues. A cephalometric radiograph (figure 1) is a twodimensional X-Ray image of the sagital skull projection [1][2]. It is used to evaluate these relationships. Cephalometric image landmarks are bony landmarks and are first located on the radiograph. Distances and angles among these landmarks are compared with normative values to diagnose a patient's deviation from ideal form and to evaluate craniofacial growth characteristics, skeletal and dental disharmonies. It is also used to evaluate results and stability of various treatment approaches. This task is challenging and has been the subject of previous research [3][4]. Our goal is the realization of a computer vision system to obtain an objective and reproducible cephalometric analysis. Indeed, large inter-expert and intra-expert variability has been noticed [2]. The main source of errors is the precise identification of landmarks. The two main causes are the subjectivity in the interpretation of the landmark definitions and the positional repeatability of human experts. Landmarks are difficult to distinguish on images and interpretation needs a long training time. In a computerized method, the formal descriptions of landmarks used by clinicians are not directly transposable: we then use a three step approach: the first step provides an initial estimation of the landmark positions, using statistical models and training sets. In a second step, a Region Of Interest (ROI) is computed using training sets and initial estimations. The third step fits specific models to the given cephalogram in the previous ROI.

Because of large variability of the shape and the morphology of human head, large variation of spatial coordinates of landmarks are observed and must be reduced. The main feature of this work is the registration of these coordinates in an adaptative way, i.e. in a coordinates space which takes into account the morphology of each individual. In this space, variability of these landmarks is greatly reduced and a small area for each landmark can be automatically defined on each cephalogram. In this ROI, the probability to find the associated landmark is $99.5 \%$.

The anatomical definition of landmarks is often the intersection of two bones or sutures. Bones and sutures are thin dark or white lines on the image. For each ROI, a specific process is defined to detect each bones using

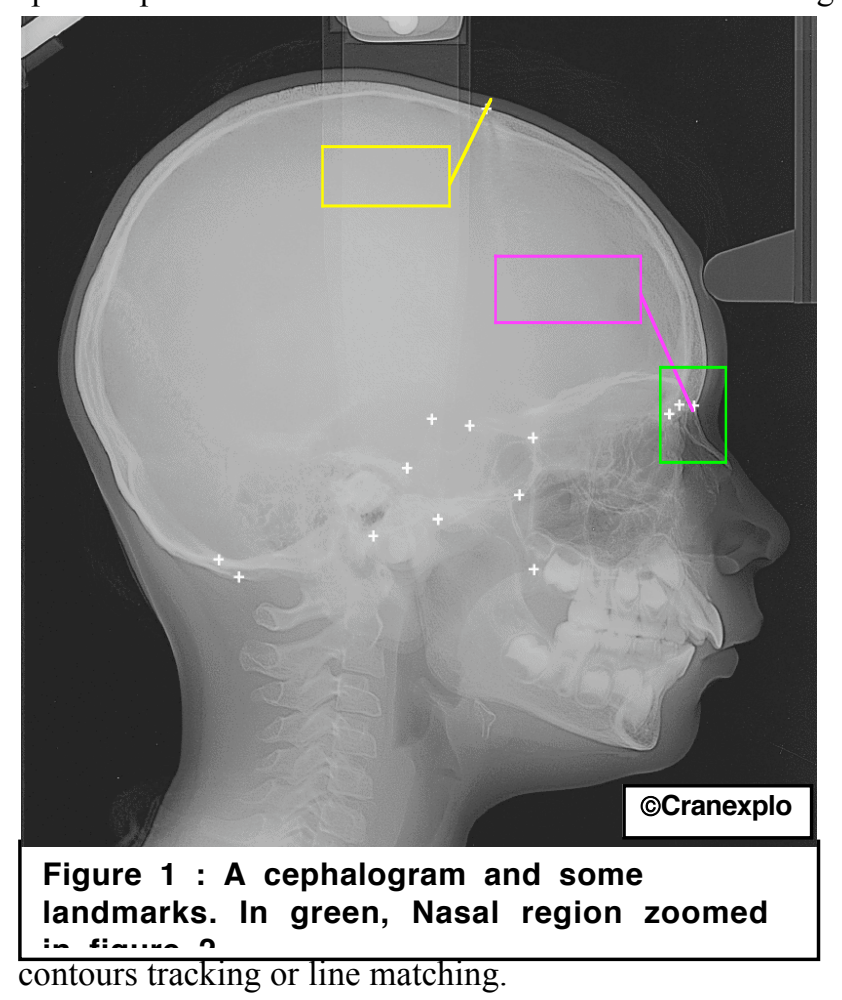

\section{Method}


To compare spatial coordinates of a deformable model, model and image must be registered in a common coordinates space. This coordinates space must be computed for each new cephalogram. Several other works use registration (rigid, affine or elastic) to superimpose or to deform one image on another one [7], one image on an atlas [6] or a set of landmarks on another one [5]. Because of the large variability of the head, rigid and even affine registration methods have a poor accuracy [7]. Elastic registration methods are CPU time consuming and are based upon fluid or solid deformation theory [8]. In our case, the head is composed of several solid parts (bone) which are fitted into together. Then, fluid or solid deformation theory is not adequate. We use a feature which summarizes global and affine registration and local elastic deformation of each individual: the sampled endocranial contour. In a first part, we describe the coordinate space in which all further process will take place. In a second part, our model is described. The key feature of the model is to store the variability of the features derived from the sampled endocranial contour in the previous coordinate space. The third part explains how the model is used to compute the desired landmarks on a new radiography: the mathematical formulation shows that a simple least square inversion is needed to compute the position of the landmarks. The fourth part explains how the ROI are automatically defined from the analysis of the error between the estimated and the real locations. The fifth part describes two examples of the identification process in each ROI.

\subsection{Adaptative and individual coordinate space}

To reduce the variability of the location, an adaptative coordinates space is automatically computed for each cephalogram, in order to reduce the influence of the shape and morphology of the patients. This is obtained by using the internal surface of the skull (endocranial contour) which is easily detected on lateral projection using edges detectors [9] and active contours [10]. This curve is sampled (n points $P_{i}$ on the curve). The origin of the sampling is given by the point $P_{0}$ with the higher curvature, i.e. a point near the intersection of the curve and of the nasal bone.

Let the set of vectors $R$ be defined by each pair of points $P_{i}$ and $P_{j}$ of the sample endocranial contour.

$R=\left\{\left(O_{i}, \vec{v}_{i}\right) \forall(j, k) \in\{1 \ldots n\} j<k, \exists i / O_{i}=P_{j}\right.$ and $\left.\vec{v}_{i}=\vec{P}_{i} P_{k}\right\}$

Let $M\left(M_{x}, M_{y}, 1\right)$ be a point. The coordinates $\alpha_{\imath}$ of $\mathrm{M}$ in $\mathrm{R}$ are defined by the scalar product (or projection) of $\mathrm{M}$ and each vectors of R i.e. $\alpha_{i}=\left\langle\underset{O_{i} M}{\rightarrow} \mid \vec{v}_{i}\right\rangle$

Then, we can write under the matricial form :

$$
\alpha_{i}=\left(\begin{array}{lll}
v_{i x} & v_{i y} & -\left(O_{i x} v_{i x}+O_{i y} v_{i y}\right)
\end{array}\right)\left(\begin{array}{c}
M_{x} \\
M_{y} \\
1
\end{array}\right)=(A)\left(\begin{array}{c}
M_{x} \\
M_{y} \\
1
\end{array}\right)
$$

The coordinates $\alpha_{i}$ are the projection of the point $M$ on each vector built using the endocranial contour. As this contour evolves with the shape of the head of the patient, the coordinates of the point $M$ in this space evolve with the morphology of the patient: this is our adaptative coordinates space.

\subsection{Model}

Our model represents the position and the variability of these positions by observing the location of the landmarks drawn by an expert on a training set of 28 cephalograms. The mean $\mathrm{E}(\alpha)$ and the standard deviation $\sigma(\alpha)$ of the location of each landmark are computed and stored in this coordinates space. As the number of coefficients $\alpha_{i}$ is $n(n+1) / 2$, and because all the coefficients are not relevant for each point $X$ in the image, we have estimated the number of coefficients which are really useful. This number has been estimated by calculating the evolution of the mean error on the training set versus the number of coefficients. We have observed a minimum value of the error between 18 and 22 coefficients and we have kept 20 coefficients. In this model, spatial relationships between landmarks are not explicitly computed. In fact, the quantitative evaluation of these relations is the goal of the cephalometry, to determine the abnormality of some dental position. We notice that using the endocranial contour, spatial relationships are indirectly represented in the model and used in the statistical landmarking of new cephalograms.

\subsection{Statistical estimation of landmarks}

To determine the unknown position of the landmark $X$ in a new cephalogram, we can write:

$$
E[\alpha]=A . X
$$

where $\mathrm{A}$ is computed by detecting the endocranial contour and the vector $\alpha$ on the new cephalogram.

$X$ can be computed with a least square inversion matrix, using the constraint $X_{z}=1$. We have used a weighting matrix: the importance of each coefficient $\alpha_{i}$ is proportional to its confidence, i.e. inversely proportional to the standard deviation ( $P$ matrix).

$$
P=\left(\begin{array}{cccc}
\frac{1}{\hat{\sigma}_{0}} & 0 & \cdots & 0 \\
0 & \frac{1}{\hat{\sigma}_{1}} & \ddots & \vdots \\
\vdots & \ddots & \ddots & 0 \\
0 & \cdots & 0 & \frac{1}{\hat{\sigma}_{p}}
\end{array}\right)
$$


The cost function in the least square inversion becomes:

$$
J=\|P \hat{E}[\alpha]-A P X\|^{2}
$$

The estimated initial position of the unknown landmark $\mathrm{X}$ is then given by the equation

$$
\hat{X}_{M C}=\left(A^{t} P^{t} P A\right)^{-1} A^{t} P^{t} \hat{E}[\alpha]
$$

Thus, on a new cephalogram, we can compute the set $R$ of vectors $V i$ and the matrix $A$ and solve the last equation, which gives the desired estimation.

\subsection{Regions of interest}

The variability of the location on this new cephalogram is given by computing the standard deviation between the predicted landmarks and the real landmarks on the training set by :

$$
\Delta X=\sum_{\text {landmarks }}\left|\hat{X}_{i}-X_{i}\right|
$$

We assume that the distribution of this error is a gaussian distribution. For each landmark, the ROI is defined by a window whose center is the estimated location of the landmark. The width and the height of the windows are equal to 3 times the standard deviation of the error $\Delta X$ along the $\mathrm{X}$-axis and $\mathrm{Y}$-axis (figure 2). This window

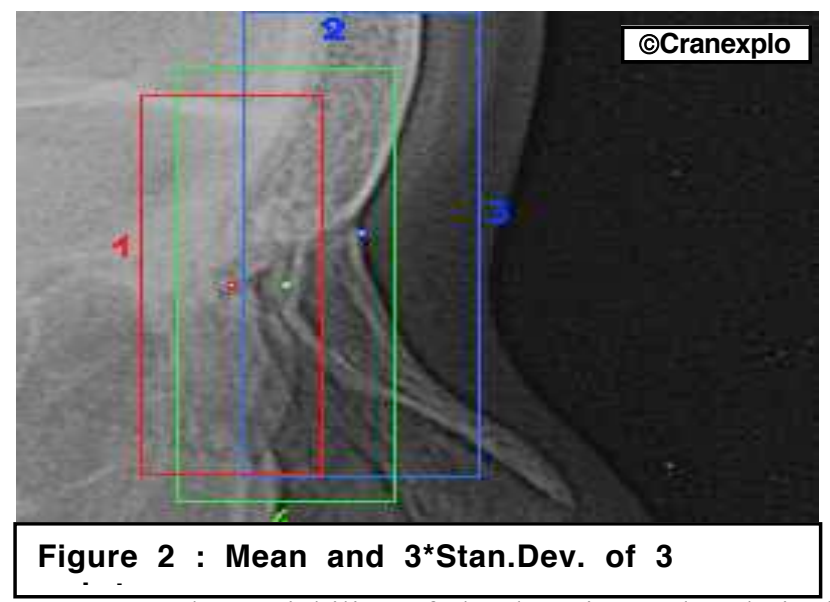

represents the variability of the location. The desired landmark is expected to be found in this area with a probability of $99.5 \%$.

\subsection{Structural identification of landmarks}

The last step is the precise location of landmarks. The automatic process tries to be a close reproduction of the anatomical definition of landmarks. All the landmarks used can be defined by an intersection of two or more structures (bone or suture). These structures are thin dark lines (suture), edges of white regions (bones) or thin white lines (thin bones) on X-Ray images. The general scheme of the precise identification process is to detect each structure involved in the definition. The intersection of these structures is then detected. As there is a lot of algorithms to detect curvilinear structures on images, the structures for each landmark are detected with specific processes using a-priori knowledge to optimize the accuracy of the detection. Two examples are described to illustrate the method.

2.5.1. Na Point. The $\mathrm{Na}$ point is defined by the intersection of the nasal bone and of the frontal bone. These two structures are easily detected on X-Ray images using a gradient operator and by tracking the maximum value of the gradient norm. The frontal bone is an edge with a high gradient value. It has an intersection with the superior horizontal limit of its Region Of Interest. The point with the highest gradient value on the top of the ROI is the origin point of the nasal bone. This first structure is found from this origin point by tracking the maximum gradient value. The nasal bone is detected using the same process, except that the origin point is located on the bottom part of the ROI. The intersection of the two bones is the Na point (blue point on figure 2).

2.5.2. Br Point. The Br point is defined (figure 3 ) by the intersection of the coronal suture and of the external limit of the skull (exocranial contour). The skull is easily detected on X-Ray images using a gradient operator. The coronal suture is a little dark line in a white bone (skull), with a $2 \mathrm{~mm}$ width ( 1 to 3 pixels) and $5 \mathrm{~mm}$ length. As the external limit of the skull can be easily detected, an intensity model (figure 4) is matched along the exocranial contour. The implementation is an iterative process. All the matched location of the intensity model along the

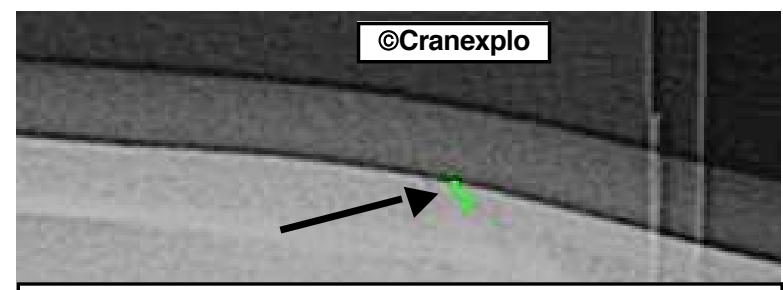

Figure 3: Br point. In green, coronal

exocranial contour are recorded. Then, the contour is displaced from one pixel toward the center of the skull using active balloons and the intensity model is matched along the new position of the contour. The matching locations are recorded if they are connected to the previously detected locations. This iterative process is stopped when the endocranial contour is reached. If several sutures are detected, orientation and length of the real suture are used to select the best candidate. 


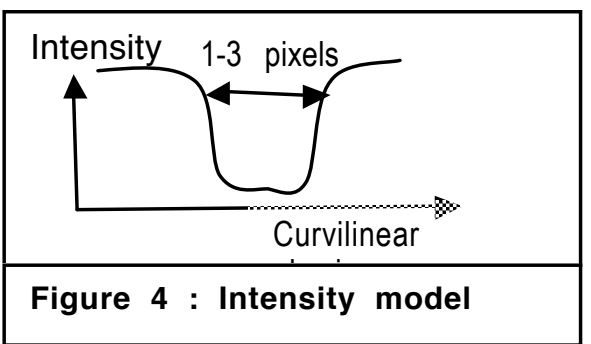

\section{Results}

The initial statistical estimation of the location of the landmarks and the ROI have been tested on 58 cephalograms. The landmarks are located in the automatically computed ROI in 57 cephalograms. The cephalogram with one error presents an abnormal cranial form, which is not the purpose of cephalometry.

The error between the real position and the estimated position of landmarks has been computed on the training set (28 cephalograms). For the landmarks near the Nasal region (figure 2), the maximum error is about $12,5 \mathrm{~mm}$, and the mean error is about $4.5 \mathrm{~mm}$. For landmarks in the middle of the skull, the maximum error is about $6 \mathrm{~mm}$ and the mean error is about $3 \mathrm{~mm}$. The influence of the shape is then better corrected near the middle of the skull. Most of the points to detect are located in this region. A second comparison can be done between our error and the variability between experts, which is greater than our error.

The evaluation of the precise location have been done by a binary test on 58 cephalograms: detected or not, i.e the anatomical definition has been respected or not. Using this criteria, the success rate is $75 \%$ for the Na point and $90 \%$ for the $\mathrm{Br}$ point. The $\mathrm{Br}$ point is not always present because this suture disappears among old children. These cases have been excluded.

\section{Conclusion}

We have presented a three step methodology to automatically label landmarks on an image.

The first and the second step automatically focuses the attention of an expert (human or computer software) on a small area where the desired landmark is expected. We have used a statistical approach, building an implicit gaussian model with a training set of labelled images. The model is simply projected onto the new image, by a generalized least square inversion to determine the position of the landmarks. The main feature of this work is that unlike traditional affine registration and mean model, we use a non linear registration before building a model, which is reduced to the mean value and standard deviation of the coordinates of the landmarks. Although the spatial relationships are not explicitly exploited in this model, the statistical locations of the landmarks are linked together by the internal surface of the skull. The third step is the precise location of each landmark, using the anatomical definition. The general scheme of the process is to find the intersection between two structures. Each landmark will use specific process to detect these structures. Two landmarks have been studied and the obtained results are quite satisfying.

Future works deal with a large database of cephalograms to optimize the statistical process. Other landmarks will be studied for precise location. As the success rate must be increased, we will define several identification processes for one landmark, which will be combined using cooperative or competition criteria.

\section{Acknowledgements}

This work is achieved with Telecrane Innovation SA.

\section{References}

[1] J.A.MacNamara : A method of Cephalometric Evaluation. American Journal of Orthodontics, 86, pp 449-469, 1984

[2] M.Cretot : L'image téléradiographique en céphalometrie, Edition CPD, 1989

[3] A.Ratter, O.Baujard, C.J.Taylor, T.F.Coostes : A distributed Approach to Image Interpretation Using Model Based Spatial Reasoning. BVMA, pp 476-481, 1993.

[4] D.N.Davis, C.J.Taylor : A Blackboard Architecture for Automatic Cephalogram Analysis, Medical Informatics, Vol 16, pp 137-149, 1991

[5] F. L. Bookstein: Principal Warps : Thin-Plate Splines and the Decomposition of Deformations, IEEE PAMI, 11, (6), pp 567-585, 1989

[6] G. Subsol, J.Ph. Thirion, N. Ayache. " A general scheme for automatically building 3D morphometric anatomical atlases: application to a skull atlas ». Medical Image Analysis, 2 (1) : pp 37-60, 1998

[7] R. Woods et al. "Automated Image Registration: I. General Methods and Intrasubject, Intramodality Validation". Journal of Computer Assisted Tomography. 22 (1): pp 139-152, 1998.

[8] G.E. Christensen, R.D. Rabbitt, M.I. Miller, Deformable Templates Using Large Deformation Kinematics, Image Processing (5), pp. 1435-1447, 1996.

[9] R. Deriche. Fast algorithms for low-level vision. IEEE Transactions on PAMI, 1(12): pp 78-88, January 1990.

[10]L.D.Cohen, I.Cohen, Finite-Element Methods for Active Contour Models and Balloons for 2-D and 3-D Images, IEEE Trans. PAMI. Vol. 15. pp. 1131-1147. 1993. 